\title{
Análise sobre Riscos Ergonômicos no profissional Docente
}

\author{
Analysis on Ergonomic Risks in the Teaching professional \\ Análisis de Riesgos Ergonómicos en el profesional Docente
}

Geovanna Renaissa Ferreira Caldas

ORCID: https://orcid.org/0000-0001-9820-309X

Centro Universitário de Juazeiro do Norte, Brasil

E-mail: geovannacaldas@hotmail.com

Eli Carlos Martiniano

ORCID: https://orcid.org/0000-0002-0546-6315

Hospital Regional do Cariri, Brasil

Hospital São Camilo, Brasil

E-mail: elimartiniano@gmail.com

Lizandra Ellem Silva De Souza

ORCID: https://orcid.org/0000-0003-2398-325X

Centro Universitário de Juazeiro do Norte, Brasil

E-mail: lizandraaellen@ hotmail.com

Natalia Alves Nunes

ORCID: https://orcid.org/0000-0002-6667-760X

Centro Universitário de Juazeiro do Norte, Brasil

E-mail: natalia_nunes22@hotmail.com

Palomma Rafaelly Teixeira Alencar

ORCID: https://orcid.org/0000-0002-2261-4252

Centro Universitário de Juazeiro do Norte, Brasil

E-mail: loma.cristo16@gmail.com

Cicero Rafael Lopes da Silva

ORCID: https://orcid.org/0000-0001-8819-5380

Centro Universitário de Juazeiro do Norte, Brasil

E-mail: rafael.lopes@unijuazeiro.edu.br

Maria Gabriela dos Santos

ORCID: https://orcid.org/0000-0002-9443-0815

Hospital da Mulher Dr. ${ }^{a}$ Nise da Silveira, Brasil

E-mail: mariagabi111@hotmail.com

Emanuel Cardoso Monte

ORCID: https://orcid.org/0000-0002-4719-7168

Centro Universitário de Juazeiro do Norte, Brasil

E-mail: emanoelcm@gmail.com

Larissa Lessa dos Santos

ORCID: https://orcid.org/0000-0002-8947-6691

Universidade Estácio de Sá, Brasil

E-mail: larissalessaa@hotmail.com

Raiane de Carvalho Machado Oliveira

ORCID: https://orcid.org/0000-0003-1971-1307

Universidade Estácio de Sá, Brasil

E-mail: raiane_live@hotmail.com

Crystianne Samara Barbosa Araújo

ORCID: https://orcid.org/0000-0002-4168-7414

Centro Universitário de Juazeiro do Norte, Brasil

E-mail: crystianne.barbosa@unijuazeiro.edu.br

Maria Elisa Regina Benjamin de Moura

ORCID: https://orcid.org/0000-0003-3145-4307

Faculdade de Medicina do ABC, Brasil

E-mail: dayseluz.dcrp@gmail.com

Laynara dos Santos Nunes

ORCID: https://orcid.org/0000-0001-5354-5563

Universidade Federal do Ceará, Brasil

E-mail: laynara.nunes@gmail.com

Maria Zenir dos Santos Nunes

ORCID: https://orcid.org/0000-0002-1772-9266

Universidade Federal do Ceará, Brasil

E-mail: mzenir.nunes@gmail.com 


\author{
Samara Atanielly Rocha \\ ORCID: https://orcid.org/0000-0002-5622-9280 \\ Faculdade de Saúde e Humanidades Ibituruna, Brasil \\ E-mail: samaraatanielly@outlook.com \\ Petrúcya Frazão Lira \\ ORCID: https://orcid.org/0000-0001-9539-066X \\ Centro Universitário de Juazeiro do Norte, Brasil \\ E-mail: petrucya.frazao@unijuazeiro.edu.br \\ Dayse Christina Rodrigues Pereira Luz \\ ORCID: https://orcid.org/0000-0002-5719-3574 \\ Centro Universitário Saúde - Faculdade de Medicina do ABC, Brasil \\ E-mail: dayseluz.dcrp@gmail.com
}

\begin{abstract}
Resumo
Analisar riscos e situações ante ergonômicas no desempenho do papel docente. Trata-se de um estudo exploratório, descritivo de abordagem quantitativa, constituído por professores de uma Instituição de Ensino Superior, do interior do estado do Ceará, como critério de inclusão encontra-se aqueles que ministram aula no curso de graduação em enfermagem. A coleta se deu através da aplicação de um questionário de elaboração própria dos autores, utilizando a Análise Ergonômica do Trabalho, em ambiente reservado, respeitando a privacidade e confidencialidade. Os dados foram agrupados e analisados através da organização de ideias centrais sobre a presença de riscos e situações ante ergonômicas e seus principais malefícios à saúde do docente. Foi possível observar que as principalmente as características ambientais, físicas e/ou as organizacionais do local de trabalho são pertencentes e relevantes para o aparecimento ou piora dos sintomas relatados pelos docentes.
\end{abstract}

Palavras-chave: Fatores de risco; Ergonomia; Docente; Ensino.

\begin{abstract}
Analyze risks and situations before ergonomic performance of the teaching role. This is an exploratory, descriptive study with a quantitative approach, consisting of teachers from a Higher Education Institution, in the interior of the state of Ceará, as inclusion criteria are those who teach classes in the undergraduate course in nursing. The collection took place through the application of a questionnaire prepared by the authors, using the Ergonomic Analysis of Work, in a private environment, respecting privacy and confidentiality. The data were grouped and analyzed through the organization of central ideas about the presence of risks and situations before ergonomic and their main harm to the health of the teacher. It was possible to observe that mainly the environmental, physical and / or organizational characteristics of the workplace are relevant and relevant to the appearance or worsening of the symptoms reported by the teachers.
\end{abstract}

Keywords: Risk factors; Ergonomics; Teachers; Teaching.

\title{
Resumen
}

Analizar riesgos y situaciones antes del desempeño ergonómico de la función docente. Se trata de un estudio exploratorio, descriptivo con enfoque cuantitativo, conformado por docentes de una Institución de Educación Superior, en el interior del estado de Ceará, como criterio de inclusión son los que imparten clases en la carrera de pregrado en enfermería. La recolección se realizó mediante la aplicación de un cuestionario elaborado por los autores, utilizando el Análisis Ergonómico del Trabajo, en un ambiente privado, respetando la privacidad y la confidencialidad. Los datos fueron agrupados y analizados a través de la organización de ideas centrales sobre la presencia de riesgos y situaciones ergonómicas y sus principales daños a la salud del docente. Se pudo observar que principalmente las características ambientales, físicas y /u organizativas del lugar de trabajo son relevantes y relevantes para la aparición o agravamiento de los síntomas reportados por los docentes.

Palabras clave: Factores de riesgo; Ergonomía; Maestros; Ensenãnza.

\section{Introdução}

Desde muito cedo foi implantado dentro da nossa sociedade a importância de cada indivíduo desenvolver um papel de acordo com o seu ambiente e os meios que se encontra durante a vida, sendo o trabalho considerado como um artifício de inclusão para o homem. Entretanto, esta fonte de inclusão tem habitualmente se alterado ao ponto de gerar formas de danificar o bem-estar físico e/ou mental dos indivíduos, isso acontece em decorrência esforços realizados durante as práticas do trabalho (Silva et al., 2018).

A ergonomia tem como premissa o estudo das relações entre o homem e seu trabalho, equipamentos, máquinas e ambientes no qual se encontra inserido. Sendo esse, um estudo utilizado para a adoção de medidas de afastamentos por riscos e 
complicações provenientes do ambiente de trabalho e dos problemas relacionados a ele. Os riscos ergonômicos são fatores que interfiram na saúde mental ou física de um trabalhador, ocasionadas por cansaço físico, cansaço mental que geram momentos de muita exaustão e momentos de falta de atenção (Duarte \& Mauro, 2010).

Estes riscos podem ser classificados em: Físicos (Temperaturas exageradas - frio ou calor, Ruídos, Vibrações, Radiações), Químicos (Agentes químicos, substâncias químicas), Biológicos (Bactérias, Vírus, Fungos, Parasitas), Ergonômicos e Psicossociais (Desorganização e problemas na gestão do trabalho), inclui-se ainda, acidentes ocasionados por falta de sinalização, uso de produtos, uso ou falta do uso de instrumentos e proteções, pouca limpeza e higienização, erro em rotulagem de produtos e problemas na utilização de máquinas (Feitosa et al., 2013).

Tais riscos também estão presentes no dia a dia envolvendo profissionais que atuam na área da educação. Por relevância de diversos fatores, como uma grande carga horária, repetitividade, posições desconfortáveis e frequentes, trabalho exaustivo e diversos tipos de estresse no ambiente de trabalho têm gerado problemas a esses profissionais. Esses aspectos direcionados a uma rotina exaustiva do profissional da área, onde a dedicação necessita ser mental e física para com os atos com o próximo, quando devido a muito esforço, suas ações podem gerar riscos para os próprios profissionais, para outros profissionais ou para os pacientes (Nazario, Camponogara \& Dias, 2017).

A capacidade física e/ou mental para o trabalho é utilizada no objetivo de enfrentar os desafios encontrados/vivenciados, sejam esses, físicos ou psicológicos, causados pelas atividades realizadas no exercer do trabalho momentaneamente e no futuro. Deve-se então, haver um equilíbrio entre as demandas ocupacionais e a saúde daquele trabalhador (Silva et al., 2018).

O ambiente de trabalho deve se definir como um ambiente saudável e livre de riscos, acidentes, doenças e/ou sofrimentos, visto que pode interferir severamente na estabilidade de saúde daquele profissional, muitas vezes gerando tais riscos degenerativos, levando ao desgaste, sofrimento no convívio de trabalho, desconforto, falta de atenção e fadiga (Castelo et al., 2018).

Deste modo, observa-se que tal estudo torna-se relevante por explorar aspectos que influenciam diretamente sobre a manutenção do organismo humano, assim, por meio do conhecimento sobre a correlação do homem e seu ambiente, torna-se possível traçar medidas interventivas que contribuam para uma melhor vivência de práticas ergonômicas.

Sendo assim, tem-se como objetivo realizar uma análise sobre os riscos e as situações ante ergonômicas identificadas no desempenho do papel docente.

\section{Metodologia}

Esta pesquisa caracteriza-se como estudo do tipo exploratório, apresenta característica descritiva, sendo desenvolvido sobre a abordagem quantitativa.

A pesquisa do tipo exploratória permite o aprimoramento de ideias, garantindo assim, um planejamento e execução da pesquisa de maneira flexível. Pode-se, por intermeio dela, aprofundar em um determinado assunto, identificando algumas das suas particularidades (Lakatos \& Marconi, 2010).

O estudo descritivo tem como finalidade delimitar as condições inerentes a alguma situação vivenciada ou apresentada, detalhando os aspectos como tempo, lugar e/ou características de um certo comportamento individual, coletivo e/ou situacional. Este tipo de estudo procura responder perguntas como: com quem, quando e onde (Lima-Costa \&Barreto, 2003).

O método quantitativo é utilizando quando há dados numéricos, apresentando-se em grandezas monetárias, físicas ou quando escalas de altitude modificam em números. Esse tipo de pesquisa permite qualificar pessoas e/ou objetos em números, sendo a etapa inicial para que sejam realizadas outras pesquisas, e só então, seja tomada alguma decisão. A partir desse tipo de 
metodologia, torna-se possível avaliar os dados obtidos com a pesquisa, através da metrológica e técnicas matemáticas, permitindo a previsão de acontecimentos com o uso dos cálculos, mas sem excluir a relevância de dados qualitativos (Silva; Lopes; Junior, 2014 \& Pereira et al, 2018).

A amostra foi constituída por professores de uma Instituição de Ensino Superior, do interior do estado do Ceará, sendo selecionados todos os docentes que ministram aula no curso de graduação em enfermagem, por maior conveniência de contato, fazendo parte todos aqueles que concordarem e responderem o questionário.

A pesquisa foi desenvolvida em uma Instituição de Ensino Superior localizada na região do Cariri, interior do estado do Ceará. Seu desenvolvimento, desde a elaboração do projeto à escrita final do manuscrito, compreendeu o período de Abril a Agosto de 2019.

A coleta se deu através da aplicação de um questionário (Quadro 1) de elaboração própria dos autores, utilizando a Análise Ergonômica do Trabalho (AET). A aplicação ocorreu em ambiente reservado, respeitando assim a privacidade dos participantes sobre toda e qualquer informação fornecida, neste momento também foi sanada qualquer dúvida que os participantes apresentaram sobre o referente estudo.

Quadro 1 - Questionário de análise ergonômica em docentes.

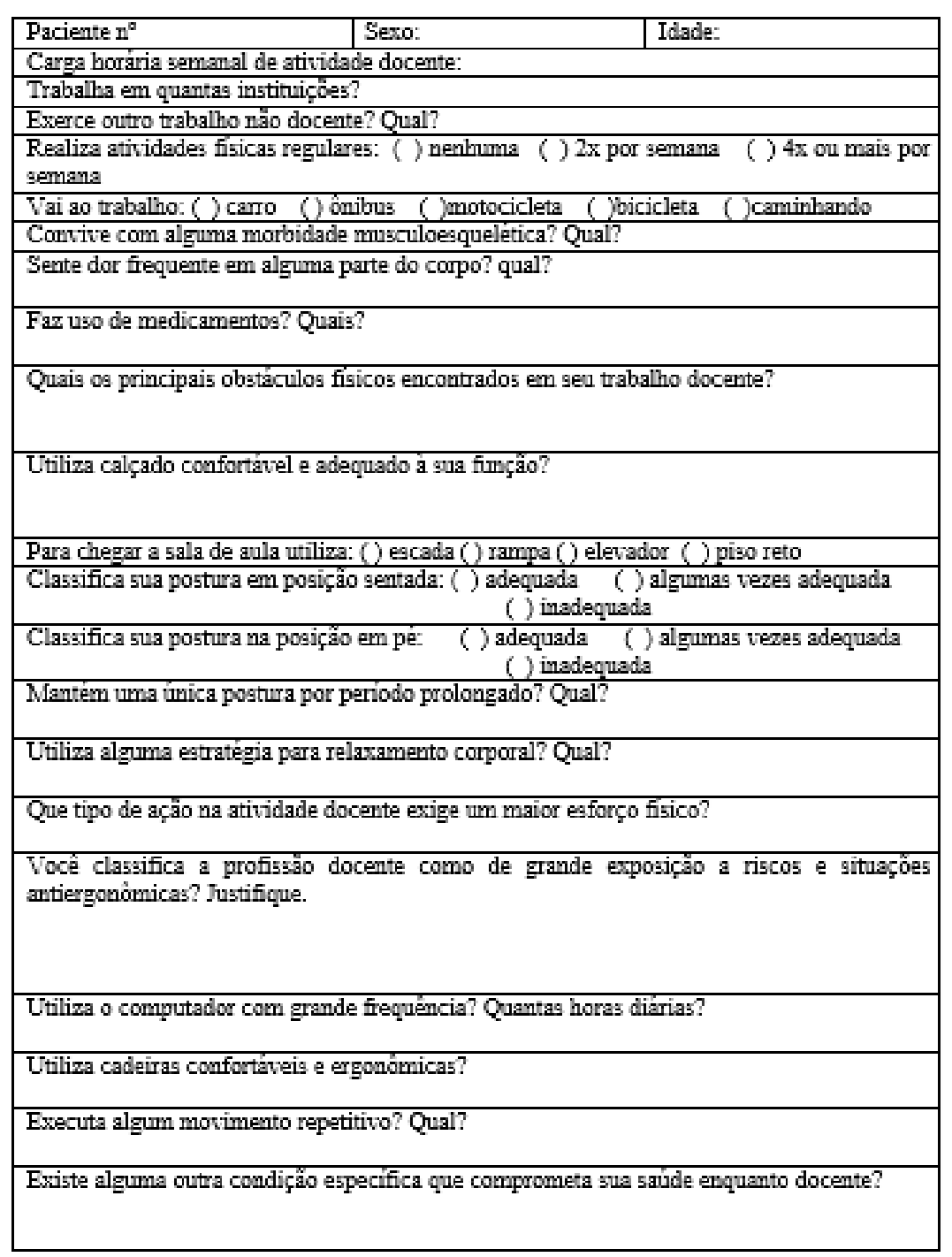

Fonte: Autores. 
Todas as informações fornecidas foram analisadas e assim sintetizadas em ideias principais conforme o foco do aspecto observado; estes aspectos foram divididos em categorias e em cada categoria apresentou-se subdivisões em grupos conforme a objetividade da resposta obtida. Cada categoria expressa as principais ideias obtidas, evidenciando indicação de que os fatores analisados apresentam relação direta com o surgimento de complicações.

Todos os participantes foram informados sobre os riscos e benefícios do presente estudo e seus objetivos; concordando com estes pontos descritos, assinaram um termo de consentimento livre e esclarecido - TCLE. Foi fornecido todas as orientações referentes à confidencialidade e sigilo de suas informações, e para a representação de suas ideias contidas no estudo não ocorreu em nenhum momento a apresentação do nome de qualquer participante. Para a validação do projeto e autorização de sua aplicação este foi enviado ao comitê de ética e a pesquisa, conforme está descrito na resolução $\mathrm{n}^{\circ} 466 / 12$ e atualizado na resolução nº 510/16 do Conselho Nacional de Saúde.

\section{Resultados}

A população de estudo, composta por 12 mulheres e 1 homem, com idade média de 41,08, com predomínio de indivíduos nas faixas etárias de 30 a 40 anos (50\%), prevalência de carga horário maior que 40 horas (53,84\%) e trabalho em 2 instituições $(53,84 \%)$.

O primeiro parâmetro avaliado foi a atividade física, onde observou-se que a maior parte dos entrevistados não realizam atividade física nenhum dia da semana $(53,84 \%)$, se deslocam para o trabalho de carro (100\%) e se deslocam para a sala utilizando a rampa $(61,53 \%)$.

Segundo parâmetro avaliado foi doenças e morbidades musculoesqueléticas, nos quais, 76,92\% não apresentam ou convivem com nenhuma doença e 53,9\% apresentam dor em alguma parte do corpo, sendo relatados locais como coluna, joelho, pé, pescoço, pernas e tudo. Foi avaliado medicamentos utilizados no dia a dia, nos quais, 69,23\% não faz uso de medicação, e os que fazem usam, relataram como principais os: Polivitamínicos, Micardis, Bupropiona, Escitalopram e Analgésicos.

No terceiro parâmetro examinou-se características da profissão. Sobre atividades que exigem esforço físico, 36,36\% relatam muito tempo em pé, mas ainda destacaram situações como uso da voz, muito tempo sentado, estágio, aulas práticas e teóricas, correções manuais. Sobre o uso de computadores, apresentaram uma média de 6,75 horas ao dia. Uso de cadeiras confortáveis, dos quais, $46,1 \%$ não utilizam. Movimentos repetitivos, sendo $61,5 \%$ de digitação e condições específicas que comprometam a saúde, onde 76,9\% não apresentando nenhum tipo de condição.

Por seguinte, foi indagado se os entrevistados consideravam a profissão docente como grande exposição a riscos ergonômicos. 46,15\% consideraram que há grande exposição, utilizando respostas como "São atividades", "Ficamos muito tempo em pé", "O professor desgasta as cordas vocais", "São muitos períodos na frente do computador", "O material do trabalho diário é pesado", "O professor utiliza bancada de laboratório e cadeiras não ergonômicas", "Jornada excessiva de trabalho", "Pouco tempo de descanso", "Muitas posições inadequadas por longos períodos".

No quarto parâmetro foi avaliado os riscos ergonômicos na profissão docente. Ao avaliar os obstáculos físicos, foi observado respostas variadas como uso de cadeiras inadequada, quadro alto, correção de provas, desgaste vocal, organização do tempo e iluminação das salas, 33,33\% não identificaram problemas no ambiente físico. Em relação a postura, a posição sentada prevaleceu inadequada $(46,15 \%)$ e na posição ortostática prevaleceu algumas vezes adequada $(53,84 \%)$.

No quinto parâmetro foi questionado sobre conforto. No quesito calçados, $69,23 \%$ utilizam modelos confortáveis. E sobre uso de técnicas para relaxamento, 53,84\% não utilizam nenhum tipo de método relaxante, mas foram citados práticas como pilates, academia, música, yoga, meditação e massagens. 


\section{Discussão}

De acordo com os parâmetros utilizado foi possível analisar as principais dificuldade desses profissionais frente suas funções onde pudemos observar que existe uma carga de estresse frente a sobrecarga de atividades realizadas além do cansaço físico que é uma queixa prevalente pelos mesmos.

Segundo a pesquisa de Caran et al (2011), 58,9\% dos empregados nessa área tem mais de um vínculo institucional e que a carga horaria de trabalho chega a 40 horas semanais que contribui para o desgaste e cansaço assim sendo fatores de riscos para dificuldades de saúde. Apresentando um consenso com os dados obtidos durante a realização da nossa determinada pesquisa onde observou-se que a jornada de trabalho é um dos fatores que contribui para o aparecimento de problemas.

Entre a relação dos parâmetros analisados foi observado um agrupamento de problemáticas, como, desgaste físico, mental e carga de trabalho elevada que levam a alguns problemas de saúde e que a estruturação da instituição acaba que contribuindo para algumas delas. O que torna importante a adaptação do trabalho para essas pessoas para assim diminuir os riscos ergonômicos encontrados para assim manter a qualidade de vida dessas pessoas melhorando a rotina de trabalho.

Frente a realização desse estudo, foi possível observar que algumas pessoas buscam terapias alternativas que favorecem para diminuição das problemáticas citadas como massagem, terapia, uso de medicamentos e educação física, mas são poucos os que fazem uso dessas práticas.

Silva et al (2016) aponta que existem docentes que fazem uso de atividades preventivas individuais que contribuem para o enfrentamento das problemáticas encontrada na profissão: o uso de exercícios regulares, alongamentos diários entre outros, mas que essas práticas ainda não são comuns no ambiente universitário. Vendo que em nosso estudo foi possível observar que 53,84\% dos pesquisados não fazem uso de nenhum método para relaxamento.

Um dos fatores mais citados foi o desgaste da voz que é um dos problemas mais encontrados entre essa profissão, pois utilizam muito a voz para ministrar as aulas e muitas vezes precisam aumentar a intensidade da voz na tentativa de vencer os ruídos encontrados no ambiente de trabalho o que faz com que fortifique os problemas vocais assim trazendo ainda mais prejuízos.

Segundo Valente, Botelho e Silva (2015), existem outros fatores de risco que podem ocasionar no desgaste da voz, como a umidade da sala de aula, presença de poeira do giz e falar em excesso onde o professor é vulnerável a essa problemática pois está em constante contato com elas. Onde cita que o principal fator é o estresse pois está ligado a outros riscos encontrados.

Os resultados foram provenientes na interpretação frente aos meios adquiridos por profissionais em relação aos riscos ergonômicos sofridos, principalmente no convívio do docente. Análise foi explorada por meio da metodologia qualitativa, permitindo visões sobre os trabalhadores em relação aos riscos presentes na profissão.

Em um dos parâmetros referentes ao questionário que expõem o esforço, em relação à profissão da docência, obtivemos quantidade relevante quanto ao tempo em pé e desgaste vocal. Esse fato tem acontecido com frequência e pode estar ligada a carga horária extensa de cada profissional, já que obtivemos prevalência superior a 40 horas. Podemos observar algumas outras abordagens que podem despertar sentimentos negativos nos trabalhadores como: movimentos repetitivos, má postura, muitas atividades, longos períodos na frente do computador, material didático pesado, utilizar cadeiras não ergonômicas e jornada excessiva.

Uma questão apontada é que a diminuição da exposição a riscos ergonômicos é favorável para os participantes dessas práticas diárias como citato por Costa e Sant'ana (2017), o qual traz com ele a expressão do sentimento de autoconfiança dos talhadores que trabalham com a ausência desses riscos. O que faz necessário a adoção de medidas que diminuam esses riscos para que torne mais fácil e traga menos prejuízos para as pessoas que vivenciam essas rotinas. 
Como fator de riscos relacionados ao ambiente físico, obteve taxa relevante em iluminação e estrutura do quadro branco, onde as unidades devem se mostrar proveniente ao bom desempenho garantindo a qualidade em base do profissional. Para intervenções educativas e fundamentais para a qualidade de vida um processo autoeducativo, referente à melhoria no quadro de dificuldade enfrentada por eles nos meios da docência. Quanto ao autocontrole como proposto na diminuição dos riscos, e indicada adaptação na carga horária, compensa e justa como redução de sobrecarga de trabalho.

Segundo Nunes, Chequer e Lacerda (2018), os fatores que podem contribuir em estresse ambientais estão dispostos nos próprios profissionais que devem atender métodos de "postura correta" em sala de aula na utilização do recurso quadro branco, em termos de iluminação, requer um consenso, embora os profissionais tenham uma porcentagem de 33,33\% em relação a má iluminação do ambiente de trabalho. Em relação aos métodos propostos aos docentes é essencial a identificação de maneira mais específica sobre os potentes riscos enfrentados, desenvolvendo estratégias para a situação ser corrigida.

Os profissionais devem ser orientados a estimar um tempo em cada posição que gere fadiga no ambiente de sala de aula, adquirindo métodos que proveram uma postura adequada, preservando as articulações e se dando a neutralidade com o próprio corpo.

Salienta-se a importância de identificar ou estar em uma única postura por um determinado tempo, o que impede uma capacitação adequado do docente frente aos alunos, deve-se analisar os métodos mais relevantes para o dia-a-dia desse profissional não se tornar prejudicial, e compartilhas os conhecimentos em conforto com o próprio ambiente de trabalho.

\section{Conclusão}

De acordo com os dados obtidos foi possível concluir que a taxa percentual de docentes que apresentaram um índice de sintomas das quais afetaram a estrutura musculoesquelética e a saúde funcional mostrou-se elevado, categorizando que a grande maioria dos Docentes apresentaram um fator de risco intensificado, particularmente pelo estado ergonômico organizatório do local de trabalho.

Foi possível observar que as principalmente as características ambientais, físicas e/ou as organizacionais do local de trabalho são pertencentes e relevantes para o aparecimento ou piora dos sintomas relatados pelos docentes, considerando ainda que poucos realizam práticas para melhora da situação, proporcionando assim, um problema a longo prazo que deve receber foco e atenção especial do empregador, da empresa e dos empregados.

Espera-se que o presente estudo possa auxiliar o desenvolvimento de futuras pesquisas, sugere-se, a mesma temática voltada para os dados qualitativas, estudos com enfoque de buscar melhores na área e no ambiente de trabalho para os profissionais docentes.

\section{Referências}

Caran, V. C. S., Freitas, F. C. T., Alves, L. A., Pedrão, L. J., \& Robazzi, M. L. C. C. Riscos ocupacionais psicossociais e sua repercussão na saúde de docentes universitários. Rev. enferm. UERJ, 19(2), 255-261.

Castelo, B. O. A. L., Ribeiro, C. G., Astrês, F. M., Teles, O. G. M., \& Santiago, R. S. Presenteismo, fatores de risco e repercussões na saúde do trabalhador de enfermagem. Av. enferm. 36(1), 79-87.

Costa, E. C., \& Sant'ana, F. R. S. Jornada de trabalho do profissional de Enfermagem e fatores relacionados à insatisfação laboral. Revista Eletrônica Acervo Saúde, 9(4), 1140-1145.

Duarte. N. S., \& Mauro, M. Y. C. Análise dos fatores de riscos ocupacionais do trabalho de Enfermagem sob a ótica dos Enfermeiros. Revista Brasileira de Saúde Ocupacional, 35(121), 157-167.

Feitosa, B. C. M., Teles, O. G. M., Carmo, C. R. M. L., Dias, T. C. R., \& Viana, A. G. A. Riscos ocupacionais e problemas de saúde percebidos por trabalhadores de enfermagem em unidade hospitalar. Cienc. enferm. 19(3), 63-71.

Lakatos. E. M., \& Marconi, M. A. Fundamentos de metodologia. (3a ed.), Atlas. 
Research, Society and Development, v. 10, n. 2, e42910212716, 2021

(CC BY 4.0) | ISSN 2525-3409 | DOI: http://dx.doi.org/10.33448/rsd-v10i2.12716

Lima-Costa, M. F., \& Barreto, S. M. Tipos de estudos epidemiológicos: conceitos básicos e aplicações na área do envelhecimento. Epidemiol. Serv. Saúde, 12(4), 189-201.

Nazario, E. G., Camponogara, S., \& Dias, G. L. Riscos ocupacionais e adesão a precauções-padrão no trabalho de enfermagem em terapia intensiva: percepções de trabalhadores. Revista Brasileira de Saúde Ocupacional, 42(7).

Nunes, A. M. S., Chequer, L. O., \& Lacerda, L. Riscos ocupacionais relacionados à enfermagem no ambiente hospitalar. Rev. Educ. Meio Amb. Saú. 8(3).

Pereira, A. S., Shitsuka, D. M., Parreira, F. J., \& Shitsuka, R. Metodologia da pesquisa Científica, Universidade Federal de Santa Maria.

Silva, D., Lopes, E. L., \& Junior, S. S. B. Pesquisa quantitativa: Elementos, paradigmas e definições. Revista de Gestão e Secretariado - GeSec, São Paulo, 5(1), 01-18, jan./abr. 2014.

Silva, L. A., Fritsch, J. N. D., Rita, C. M. B., Leite, G. R., Maia, L. G., Silveira, S. E., Evangelista, R. A., \& Pelazza, B. B. Riscos ocupacionais e adoecimentos entre professores da rede municipal de ensino. Journal Health NPEPS, 1(2), 178-196.

Silva, T. P. D., Araújo, W. N., Stival, M. M., Toledo, A. M., Burke, T. N., \& Carregaro, R. L. Musculoskeletal discomfort, work ability and fatigue in nursing professionals working in a hospital environment. Rev. esc. enferm. USP, 52, 2018.

Valente, A. M. S. L., Botelho, C., \& Silva, A. M. C. Distúrbio de voz e fatores associados em professores da rede pública. Revista Brasileira de Saúde Ocupacional, 40(132), 183-195. 